\title{
Flux quantization in stationary and nonstationary states in long Josephson junctions
}

\author{
K. N. Yugay, N. V. Blinov, and I. V. Shirokov \\ Omsk State University, Omsk, 644077, Russia \\ E-mail: yugay@phys.omsu.omskreg.ru
}

Received September 17, 1999, revised June 15, 2000

\begin{abstract}
Dynamical chaos, states stability in long Josephson junctions are investigated from the point of view of the flux quantization. It is shown that the stationary Meissner and fluxon states having integer number of fluxons are stable. Stationary antifluxon states also having integer number of the flux quanta and all other states with half-integer number of flux quanta are unstable. The transitions between all states - Meissner, the states having the integer and half-integer number of the flux quanta - take place in the nonstationary case, and all these states are dynamically equivalent, but the number of the flux quanta is a nonregular time-dependent function for the chaotic states and regular for the regular ones.
\end{abstract}

PACS: 05.45.+b, 74.50.+r

\section{Introduction}

Long Josephson junctions (LJJ) are of big interest first of all from the point of view of making SQUIDs on the basis of them. However a LJJ is of interest also as a simple and at the same time deep physical and mathematical model of nonlinear phenomena. Dynamical chaos in a LJJ is a subject of intensive researches [1-19], it may be a source of the dynamical noise.

It is well known that the number of solutions of the nonlinear stationary Ferrell-Prange equation for the conventional Josephson phase variable (it is just a phase difference of the wave functions of a superconducting condensate on the junction) describing stationary states in a LJJ at the given boundary conditions depends on the value of an external magnetic field $H_{0}$, a bias current $\beta$ and a total length of the junction $L$. It is clear, that only one solution may realize in the concrete junction at given values $H_{0}, \beta$ and $L$. Evidently, this state must be one of the solutions of the Ferrell-Prange equation. Stationary states of a LJJ being the solutions of the Ferrell-Prange equation have been investigated, for example, in Ref. 20. The question is, how does a selection of this solution happen? What is the selection of this solution affected by? The problem of the selection of the solutions of the Ferrell-Prange equation are usually treated in the framework of thermodynamical approach [20].
Within such approach one assumes that just the solution corresponding to the global minimum of the thermodynamic Gibbs potential realizes at the concrete physical conditions. In our previous work [21], we have proposed another approach based on the fact that the Ferrell-Prange equation is the stationary limit of the time-dependent sine-Gordon equation describing the dynamics of a LJJ. Thus, we applied the sine-Gordon equation with the dissipation to find the dynamical criterion of the states stability. Using this criterion we have shown that not all solutions of the boundary Ferrell-Prange problem are stable, a part of them are metastable.

In the work [22], we have also shown that in a LJJ with dissipation the selection of the specific asymptotic solution (including a nonstationary one) can be affected by the form of the initial rapidly damped perturbation independently of the initial state is chaotic or not. In other words, the small rapidly damped perturbation influences essentially the asymptotic states of a LJJ even if the initial state is chaotic. This characteristic of the LJJ we have called a memory effect. Due to this effect the dynamical chaos strangly differs from the statistical one (in the case of the statistical chaos a loss of information happens during the relaxation time).

In the presence of both an external magnetic field and dc bias current, three clusters of states can exist: stationary, regular, and chaotic. With changing the parameters of the initial perturbation, the 
system realizes transitions between these three clusters of the asymptotic states [22]. In the work [23], we have shown that the parametric $\beta-H_{0}$ plane of a LJJ is separated by the bifurcation lines on the series of regions with the different number of solutions of the Ferrell-Prange equation, and along the bifurcation line that separates the region with two stationary states from the region without ones a chaos strip arises. The number of the stationary states decreases in the presence of the bias current but also at $\beta=0$ a part of the solutions are stable, others are unstable. In this work we will try to investigate these stable and unstable states in detail. We will analyze the problem from the point of view of the flux quantization. We will show that the stationary states have only zero, integer and half-integer number of flux quanta, and the states with half-integer number of flux quanta are always unstable. The states with the integer number of flux quanta are the fluxon and antifluxon states, and the fluxon states are stable, the antifluxon ones are unstable. Among the states with the zero number of flux quanta only the Meissner states are stable, other are unstable. We will formulate the criterion, which makes possible to define the stability of the states from the point of view of the flux quantization. We will show also that the number of flux quanta is a nonregular time-dependent function for the chaos states and that allowed states with the integer and half-integer number of flux quanta are equivalent in a certain sense in nonstationary regular and chaotic - regimes.

\section{Flux quantization for stationary states}

In contradistinction to our previous work [23], we have studied the stationary states of a LJJ in detail and have mainly given attention to the flux quantization in these states. We have solved numerically the boundary Ferrell-Prange problem:

$$
\begin{gathered}
\varphi_{x x}(x)=\sin \varphi(x)-\beta, \\
\left.\varphi_{x}(x)\right|_{x=0}=\left.\varphi_{x}(x)\right|_{x=L}=H_{0} .
\end{gathered}
$$

Here $\varphi(x)$ is the Josephson phase variable; $\beta$ is the dc bias current density normalized to the critical current density of the junction $j_{c} ; x$ is the distance along the junction normalized to the Josephson penetration length $\lambda_{J} ; H_{0}$ is the external constant magnetic field perpendicular to the junction and normalized to $\tilde{H}=\Phi_{0} /\left(2 \pi \lambda_{J} d\right) ; \Phi_{0}$ is the flux quantum; $d=2 \lambda_{L}+b, \lambda_{L}$ is the London penetration length, $b$ is the thickness of a dielectric barrier, $L$ is the total length of the junction normalized to $\lambda_{J}$.
Equation (1) with boundary condition (2) has been solved numerically at different values of $H_{0}, \beta$ and $L$. The distribution of the magnetic field $H(x)=\varphi_{x}(x)$ and the current density $j(x)=\varphi_{x x}(x)$ in the junction have been calculated.

We have also calculated the thermodynamic Gibbs potential for an each state using the following equation:

$G=\int_{0}^{L}\left[\frac{1}{2} \varphi_{x}^{2}(x)+1-\cos \varphi(x)-\beta \varphi(x)-H_{0} \varphi_{x}(x)\right] d x$,

where $G$ is the thermodynamic Gibbs potential per unit length of the junction normalized to the value $\tilde{G}=\Phi_{0}^{2} /\left(16 \pi^{3} \lambda_{J} d\right)$. It should be noted that all solutions of the Ferrell-Prange equation are extremals of the functional Eq. (3). Furthermore, it is easy to show (see, for example, Ref. 24) that these extremal values correspond to the minima of the thermodynamic potential $G$.

As an illustration, the results of the calculations for $H_{0}=1.9, L=10$ and $\beta=0.06, \beta=0.08$ are listed in Tables 1 and 2.

Table 1

Stability, transitions, number of flux quanta, sort of state. $H_{0}=1.9, L=10, \beta=0.06$

\begin{tabular}{c|l|c|c|c|c}
\hline \hline $\begin{array}{c}\text { Number } \\
\text { of state }\end{array}$ & Stability & $G$ & $\begin{array}{c}\text { Transi- } \\
\text { tions } \\
k \rightarrow l\end{array}$ & $\begin{array}{c}\text { Number } \\
\text { of flux } \\
\text { quanta }\end{array}$ & $\begin{array}{c}\text { Sort of sta- } \\
\text { ble states }\end{array}$ \\
\hline \hline 1 & unstable & -12.849 & $1 \rightarrow 7$ & 2 & 2-antifluxon \\
2 & unstable & -12.912 & $2 \rightarrow 9$ & 1.5 & \\
3 & unstable & -7.592 & $3 \rightarrow 5$ & 1 & 1-antifluxon \\
4 & unstable & -7.594 & $4 \rightarrow 5$ & 0.5 & \\
5 & stable & -7.829 & $5 \rightarrow 5$ & 0 & Meissner \\
6 & unstable & -7.828 & $6 \rightarrow 5$ & 0.5 & \\
7 & stable & -13.652 & $7 \rightarrow 7$ & 1 & 1-fluxon \\
8 & unstable & -13.368 & $8 \rightarrow 7$ & 1.5 & \\
9 & stable & -17.556 & $9 \rightarrow 9$ & 2 & 2-fluxon \\
10 & unstable & -17.272 & $10 \rightarrow 9$ & 2.5 & \\
\hline \hline
\end{tabular}


Table 2

Stability, transitions, number of flux quanta, sort of state. $H_{0}=1.9, L=10, \beta=0.08$

\begin{tabular}{c|c|c|c|c|c}
\hline \hline $\begin{array}{c}\text { Number } \\
\text { of state }\end{array}$ & Stability & $G$ & $\begin{array}{c}\text { Transi- } \\
\text { tions } \\
k \rightarrow l\end{array}$ & $\begin{array}{c}\text { Number } \\
\text { of flux } \\
\text { quanta }\end{array}$ & $\begin{array}{c}\text { Sort of } \\
\text { stable states }\end{array}$ \\
\hline \hline 1 & unstable & -14.747 & $1 \rightarrow 3$ & 2 & 2-antifluxon \\
2 & unstable & -14.771 & $2 \rightarrow 3$ & 1.5 & \\
3 & stable & -20.328 & $3 \rightarrow 3$ & 2 & 2-fluxon \\
4 & unstable & -20.177 & $4 \rightarrow 3$ & 2.5 & \\
\hline \hline
\end{tabular}

Now we calculate the flux in a LJJ. In the case of the infinitely long junction the following condition takes place for the stationary states: $\left.\varphi(x)\right|_{x \rightarrow-\infty}=0$, $\left.\varphi(x)\right|_{x \rightarrow+\infty}=2 \pi$ and the total flux is $[25,26]$

$$
\Phi=\frac{1}{2 \pi} \int_{-\infty}^{+\infty} \varphi_{x}(x) d x=n \quad(n=1,2,3, \ldots) .
$$

Here the flux $\Phi$ is normalized to the flux quantum $\Phi_{0}$. In the case of the junction of the finite length the situation drastically changes, because the boundary effects must be taken into account. In this case the shielding current always flows at the edge of a junction and the total flux must be calculated in the other way excluding boundary effects. Because the shielding current can't be associated with the flux quantization in a junction, we have to exclude boundary effects from the flux calculation. Then we can write down the required flux as follows:

$$
\Phi=\frac{1}{2 \pi} \int_{x_{1}}^{x_{2}} \varphi_{x}(x) d x=\frac{1}{2 \pi}\left[\varphi\left(x_{2}\right)-\varphi\left(x_{1}\right)\right],
$$

where $x_{1}$ and $x_{2}$ are the nearest points to the left and right edges, respectively, in which $j(x) \equiv \varphi_{x x}(x)=0$. In these points $j_{x}>0$ for the fluxon states and $j_{x}<0$ for the antifluxon ones. The formula (4) is a special case of (5) because $j\left(x_{1}=-\infty\right)=j\left(x_{2}=+\infty\right)=0$.

Using the definition of the points $x_{1}$ and $x_{2}$, we find that the following three sorts of states take place, in which

$$
\begin{aligned}
& \text { 1) } \Phi=0 \\
& \text { 2) } \Phi=n, \quad n=1,2,3, \ldots \\
& \text { 3) } \Phi=n / 2 \pm \arcsin \beta / \pi, n=1,2,3, \ldots
\end{aligned}
$$

We will call these states by allowed ones. The value $\Phi=0$ corresponds to the Meissner and quasiMeissner states, $\Phi=n(n=1,2,3, \ldots)$ to the fluxon and antifluxon states and $\Phi=n / 2 \pm \arcsin \beta / \pi(n=$ $=1,2,3, \ldots)$ to the all other states.

The comparison of the case, when a bias current is equal to 0 , with the case, when $\beta \neq 0$, makes us possible to say that the dc bias current takes off the degeneration of the states with half-integer number of flux quanta. The splitting value in the flux is equal to $2 \arcsin \beta / \pi$ and at $\beta=0.06$ and 0.08 (Table 1 and 2), it is equal to 0.038 and 0.050 , respectively.

We define a stability of the stationary states as following: firstly, we solve numerically the equation for time-dependent perturbations of the stationary state (this equation can be easily found from the sine-Gordon equation as a result of linearization [21]). Then, we determine stability or instability of the stationary state with respect to small perturbations calculating the Lyapunov exponent [22]. As we can see from Tables 1 and 2, the antifluxon states and states with half-integer number of flux quanta are unstable. These states are disintegrated to some stable states (Meissner or state with integer number of flux quanta).

To find the finite stable state $l$, in which the metastable state $k$ passes (the transition $k \rightarrow l$ ), we used the solution of the Ferrell-Prange equation corresponding to the state $k$ as an initial state for the nonstationary sine-Gordon equation.

As it is seen from the results of calculations listed in the Tables, the transition $k \rightarrow l$ is accompanied always by inequality $G_{k}>G_{l}(k \neq l)$, where $G_{k}$ and $G_{l}$ are the thermodynamic Gibbs potentials of $k$ and $l$ states, respectively. If the state $k$ is stable, $k=l\left(G_{k}=G_{l}\right)$. One of the unexpected results is that the metastable, i.e., unstable, state has the thermodynamic Gibbs potential that is not necessarily more than this one for some stable states. In Fig. 1, the schemes of the transitions between states are shown at $H_{0}=1.9, L=10$, and $\beta=0.06$ (Fig. $1, a$ ) and 0.08 (Fig. 1,b). The horizontal lines correspond to the certain values of the thermodynamic Gibbs potential of states at the given parameters $H_{0}, L$ and $\beta$. As it is seen from Fig. 1, $G_{10}<G_{5}$ and $G_{10}<G_{7}$, the states 5 and 7 are stable.

Our calculations show that the number of stable states decreases with the increasing of $\beta$. For example, four stable states exist at $H_{0}=1.9, L=10$ and $\beta=0$ : the Meissner (M), one-fluxon (1f), twofluxon (2f) and three-fluxon (3f) states; we have 3 


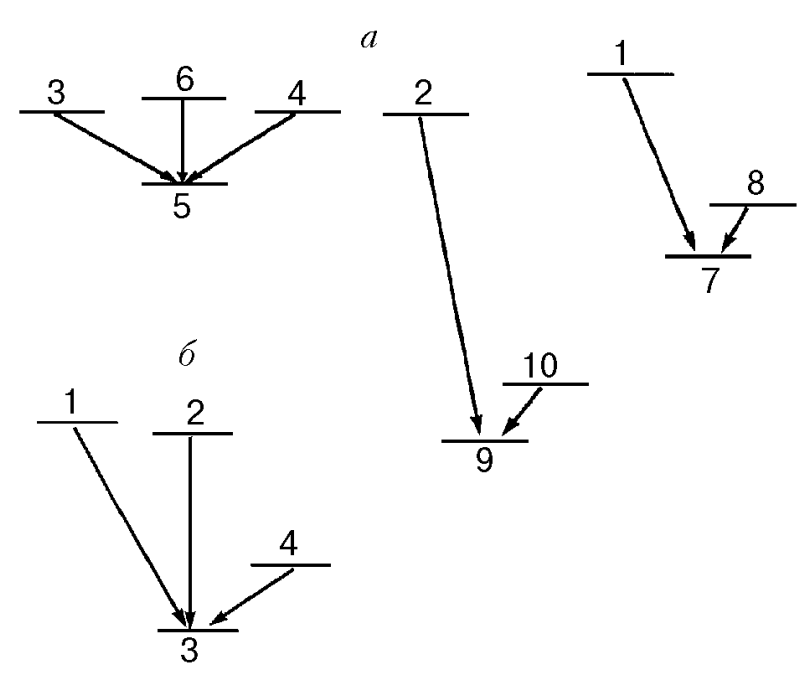

Fig. 1. The schemes of transitions between states at $H_{0}=1.9$, $L=10$, and $\beta=0.06(a)$ and $\beta=0.08(b)$.

stable states at $\beta=0.06$ : $\mathrm{M}, 1 \mathrm{f}$ and $2 \mathrm{f}$; two states at $\beta=0.07$ : $1 \mathrm{f}$ and $2 \mathrm{f}$, and one $2 \mathrm{f}$ state at $\beta=0.08$. There are no stationary states at $\beta=0.15$. The stable states with $G_{l}>G_{l_{0}}$, where $G_{l_{0}}$ corresponds to the global minimum, fall into the state with $G_{l_{0}}$ at increasing $\beta$.

The character of the stationary states changes simultaneously with the changing of the number of states when an external magnetic field $H_{0}$ is changed at other fixed parameters. For example, the number of the stable states equals to three: $M$, 1f and $2 \mathrm{f}$ at $\beta=0, L=10$ and $H_{0}=1.4$; there are two stable states: $\mathrm{M}$ and $1 \mathrm{f}$ at $H_{0}=0.6$; only one stable stationary state exists: $\mathrm{M}$ at $H_{0}=0.05$; there are three stable states again: 1f, $2 \mathrm{f}$ and $3 \mathrm{f}$ at $H_{0}=$

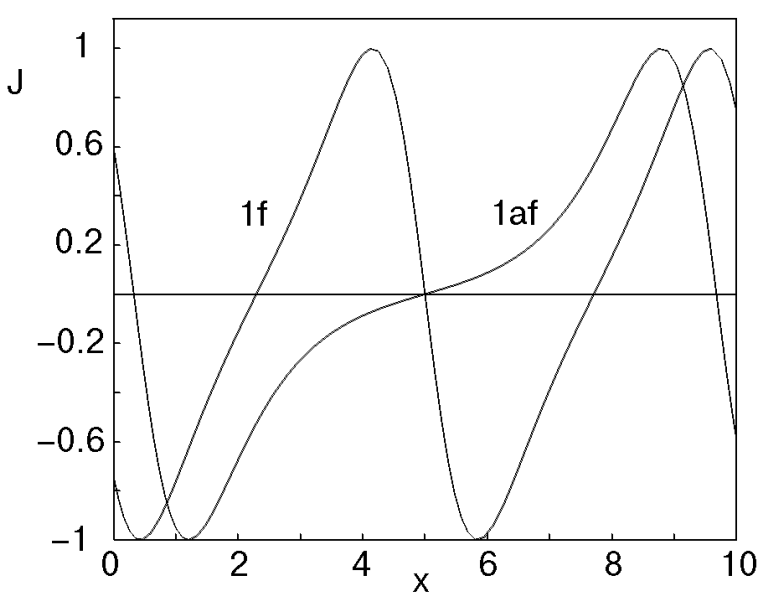

Fig. 2. Distribution of the current in one-fluxon $1 \mathrm{f}$ and one-antifluxon 1af states. $H_{0}=1.9, \beta=0, L=10$.
$=2.0$; and we have two stable states at $H_{0}=2.1: 2 \mathrm{f}$ and $3 \mathrm{f} ; 3 \mathrm{f}$ and $4 \mathrm{f}$ at $H_{0}=2.2 ; 4 \mathrm{f}$ and $5 \mathrm{f}$ at $H_{0}=2.9$.

In Fig. 2 one-fluxon (1f) and one-antifluxon (1af) states are shown at $H_{0}=1.9, \beta=0$ and $L=$ $=10$. In Tables 1 and 2 the numbers of flux quanta for stationary states are listed. The calculations allow to make the following conclusions: 1) stationary states at the given set of the parameters can have a number of flux quanta being equal to zero, half-integer and integer numbers; 2) all the fluxon and antifluxon states have the positive integer number of the flux quanta according to the criterion (5) formulated above. All fluxon states are stable and all antifluxon states are unstable; 3) all states with half-integer number of fluxon are always unstable; 4 ) in the Meissner states $x_{1}=x_{2}$ always and $n=0$. This state is stable. Besides the stable Meissner states, unstable states with $n=0$ exist, one of them is a quasi-Meissner state with $x_{1}=x_{2}$. In Fig. 3 the Meissner and quasi-Meissner states are shown for $H_{0}=1.2, \beta=0$, and $L=10$. All the quasi-Meissner states in Fig. 4 fall into some stable states.

All states with half-integer number of the flux quanta and antifluxon states are nonphysical because they disintegrate and therefore can not be realized in real systems. As to the quasi-Meissner states, all of them arise in the capacity of some intermediate states and finally disintegrate.

The number of the fluxon and antifluxon states decreases at $\beta>0$. For example, there are four fluxon and antifluxon states at $H_{0}=1.9, L=10$ and $\beta=0.06$ (instead of five ones at $\beta=0$ ), and their number equals only two at $\beta=0.08$ (one of them is two-fluxon state, other is two-antifluxon one).

Thus, not all states having the integer number of flux quanta are stable but only the fluxon states. The criterion (5) formulated above may also serves

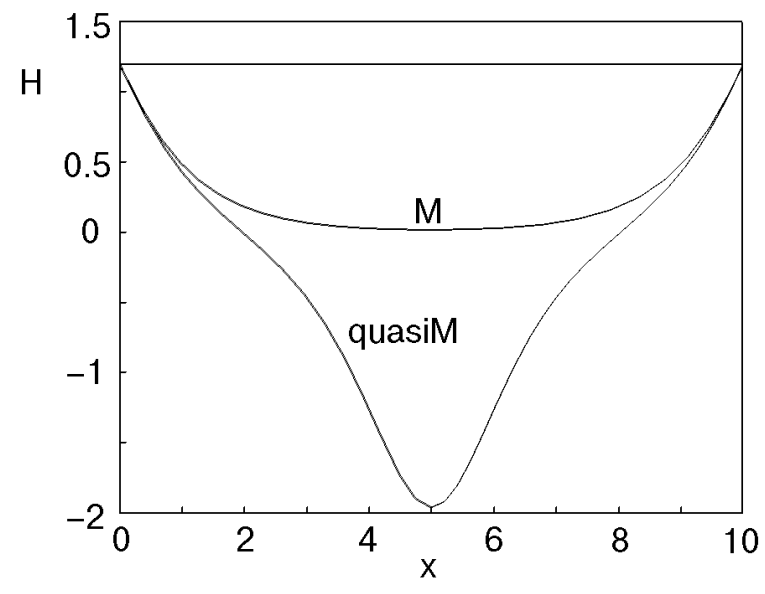

Fig. 3. Meissner and quasi-Meissner states at $H_{0}=1.2, \beta=0$, $L=10$. 
as a simple criterion of stability of the solution of the Ferrell-Prange equation.

\section{Flux quantization and chaos}

As it is noted above, the number of the stationary states decreases when dc bias current increases and, at the same time, the number of the nonstationary states increases. In general case, three clusters of states - stationary, regular and chaotic take place in a LJJ [21]. In the present Section we will consider the nonstationary regular and chaotic states from the point of view of a flux quantization.

The nonstationary states can be found by the numerical integration of the nonstationary sineGordon equation

$$
\varphi_{t t}(x, t)+2 \gamma \varphi_{t}(x, t)-\varphi_{x x}(x, t)=-\sin \varphi(x, t)+\beta
$$

with the following boundary conditions:

$$
\left.\varphi_{x}(x, t)\right|_{x=0}=\left.\varphi_{x}(x, t)\right|_{x=L}=H_{0},
$$

where $t$ is the time normalized to the inverse of the Josephson plasma frequency $\omega_{J}, \omega_{J}=$ $=\left(2 \pi c j_{C} /\left(C \Phi_{0}\right)\right)^{1 / 2}, C$ is the junction capacitance per unit area; $\gamma$ is the dissipative coefficient per unit area, $\gamma=\Phi_{0} \omega_{J} / 4 \pi c R j_{C}, R$ is the resistance of the junction per unit area. Of course, the solutions of the Ferrell-Prange Eqs. (1), (2) coincide with the asymptotic solutions of the sine-Gordon Eqs. (7), (8). All solutions of the Ferrell-Prange equation, i.e., the stationary solutions, appear from any initial state during the evolution governing by the sine-Gordon Eqs. (7), (8) but these solutions are not equivalent: some of them are stable, others are unstable (metastable).

As well as for the stationary states of the junctions of finite length, in the nonstationary case the shielding currents flowing at the junction edges must be taken into account and we define the points $x_{1}$ and $x_{2}$ again. In this case i) the locations of these points will depend on a time: $x_{1}=x_{1}(t)$ and $x_{2}=$ $=x_{2}(t)$ and ii) we must find these points using the conditions of vanishing of the total current superconducting and quasiparticle - in the junction, i.e., $j\left(x_{1}\right)=j\left(x_{2}\right)=0$, where $j\left(x_{1}\right)=\left[\varphi_{t t}(x)+\right.$ $+2 \gamma \varphi_{t}(x)-\left.\varphi_{x x}(x)\right|_{x=x_{1}}$. The same we have for the $j\left(x_{2}\right)$. This condition defines the allowed states in which
1) $\Phi=0$
2) $\Phi=n(t), n=1,2,3, \ldots$,
3) $\Phi=n(t) / 2 \pm \arcsin \beta / \pi, n=1,2,3, \ldots$

Here $n$ is a discrete function of time in the sense that it performs jumps remaining constant between these jumps. Of course, the flux $\Phi$ behaves in the same manner. In contrast to the stationary states (see (6)), the flux $\Phi(t)$ takes the different values for all the series 1)-3) in (9), spending in each of them certain time. Besides, the points $x_{1}$ and $x_{2}$ move along the junction and a character of this motion are different for regular and chaotic states.

In Figs. 4, $a$ and $4, b$ the dependences of $\Phi(t)$ for the two regimes - regular at $H_{0}=1.9, \beta=0.1$, $L=10$ (Fig. 4, $a$ ) and chaotic at $H_{0}=1.9, \beta=$ $=0.125, L=10$ (Fig. 4,b) are shown. A character of these regimes was determined by the calculation of the Lyapunov exponent $\lambda$ (see Ref. 22): in regular regimes $\lambda=0$ and in chaotic ones $\lambda>0$. The dependence of the flux in the regular regime, for example, has a regular character, and a flux evolution consists of the regular transitions from one
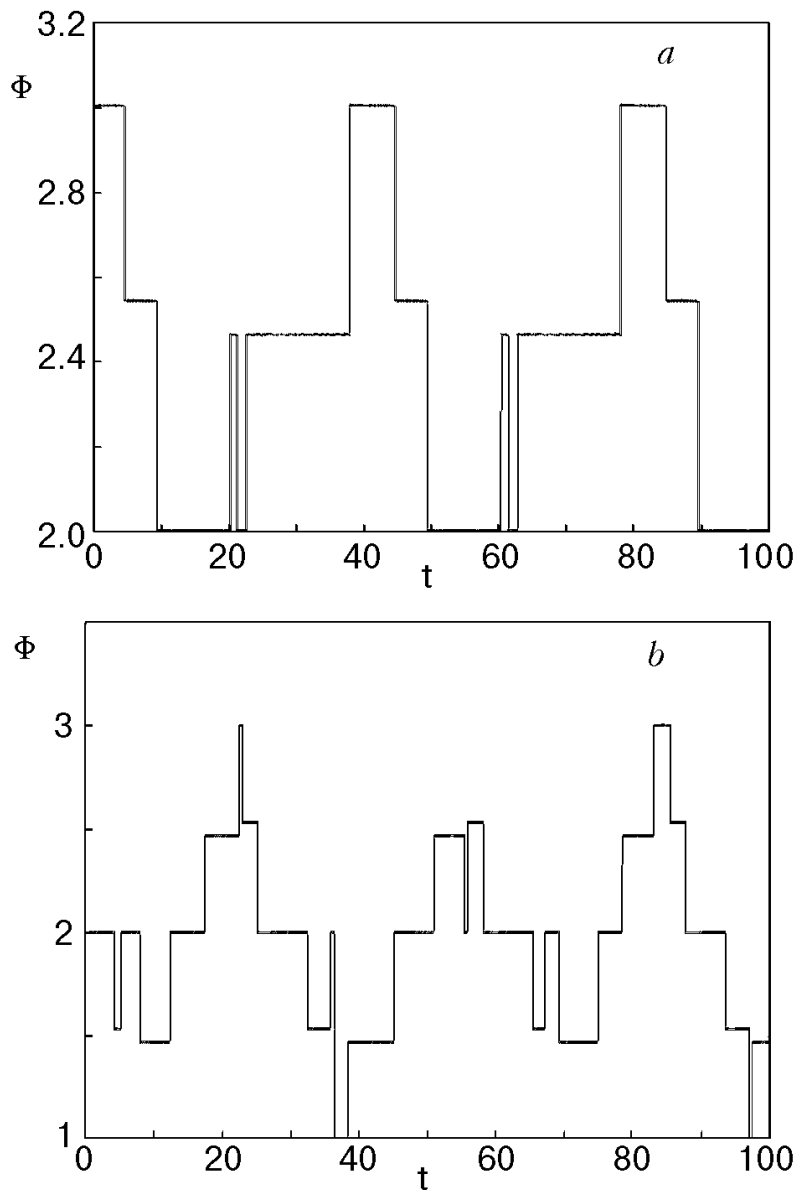

Fig. 4. Dependences of the flux $\Phi$ on time in the regular regime at $H_{0}=1.9, \beta=0.1, \gamma=0.26, L=10(a)$ and in the chaotic regime at $H_{0}=1.9, \beta=0.125, \gamma=0.1, L=10(b)$. 
allowed state to another. Of course, the dependences of $x_{1}(t)$ and $x_{2}(t)$ have the same character. In contrast, in the chaotic regime $x_{1}(t)$ and $x_{2}(t)$ are the nonregular time-dependent functions. The function $\Phi(t)$, as it is obviously seen in Fig. $4, b$, reveals the jumps between the allowed states as well as in the regular states but the response time in a fixed state changes irregularly now. We note also, that there are not any explicit preference of the states with the integer number of flux quanta in comparison with the states with the half-integer number of the flux quanta both for the regular and chaotic regimes.

So, while in the stationary regime the states with the integer number of the flux quanta (fluxon states) are prefered in the sense that they are stable, in the nonstationary regimes - regular and chaotic - the allowed states with the integer and half-integer number of flux quanta are in a certain sense equivalent.

\section{Conclusions}

In the present work we consider the problem of the states stability and the chaotic dynamics in the LJJ from the point of view of the flux quantization. We have shown that just the stationary states with integer number and half-integer number of the flux quanta take place and among them only the Meissner and fluxon states are stable, the quasi-Meissner and antifluxon states are unstable, although they have also the integer number of the flux quanta. All the other states with the half-integer number of the flux quanta are always unstable. Thus, the stable states are the states in which the flux is always quantized.

The states with the half-integer number of the flux quanta are doubly degenerate. The dc bias current removes this degeneracy. Among the stationary states just the fluxon states with the integer of the flux quanta and the Meissner state has preference, they are allowed ones.

All the allowed states with integer and half-integer number of flux quanta are equivalent in a certain sense in the nonstationary case, and a system passes from one allowed state to another, and the response time in the states is chaotic in the chaotic states and regular in the regular ones. In other words, the half-integer quantum numbers are added to the integer quantum numbers in the nonstationary - regular and chaotic - states.

1. M. Cirillo and N.F. Pedersen, Phys. Lett. A90, 150 (1982).

2. N. F. Pedersen and A. Davidson, Appl. Phys. Lett. 39, 830 (1981).

3. P. S. Lomdahl, O. H. Soerensen, and P. L. Christiansen, Phys. Rev. B25, 5737 (1982).

4. R. L. Kautz, IEEE Trans. Magn. 19, 465 (1983).

5. A. H. MacDonald and M. Plischke, Phys. Rev. B27, 201 (1983)

6. M. P. Soerensen, N. Arley, P. L. Christiansen, R. D. Parmentier, and O. Skovgaard, Phys. Lett. 51, 1919 (1983).

7. A. R. Bishop, K. Fesser, P. S. Lomdahl, and S. E. Trullinger, Physica D7, 259 (1983).

8. J. C. Eilbeck, P. S. Lomdahl, O. H. Olsen, and M. R. Samuelsen, J. Appl. Phys. 57, 861 (1985).

9. B. S. Han, B. Lee, O. G. Symko, W. J. Yeh, and D. J. Zheng, IEEE Trans. Magn. MAG-25, 1396 (1989).

10. W. J. Yeh, O. G. Symko, and D. J. Zheng, Phys. Rev. B42, 4080 (1990).

11. G. F. Eriksen and J. B. Hansen, Phys. Rev. B41, 4189 (1990)

12. X. Yao, J. Z. Wu, and C. S. Ting, Phys. Rev. B42, 244 (1990).

13. L. E. Guerrero and M. Ostavio, Physica B165-166, 11657 (1990).

14. I. H. Dalsgaard, A. Larsen, and J. Mygind, Physica B165166, 1661 (1990).

15. S. Rajasekar and M. Lakshmanan, Physica A167, 793 (1990).

16. S. Rajasekar and M. Lakshmanan, Phys. Lett. A147, 264 (1990).

17. N. Gronbech-Jensen, P. S. Lomdahl, and M. R. Samuelsen, Phys. Rev. B43, 1279 (1991).

18. N. Gronbech-Jensen, Phys. Rev. B45, 7315 (1992).

19. J. McDonald and J. R. Clem, Phys. Rev. B56, 14723 (1997).

20. C. S. Owen and D. J. Scalapino, Phys. Rev. 164, 538 (1967).

21. K. N. Yugay, N. V. Blinov, and I. V. Shirokov, Phys. Rev. B49, 12036 (1994).

22. K. N. Yugay, N. V. Blinov, and I. V. Shirokov, Phys. Rev. B51, 12737 (1995)

23. K. N. Yugay, N. V. Blinov, and I. V. Shirokov, Fiz. Nizk. Temp. 25, 712 (1999) [Low Temp. Phys. 25, 530 (1999)].

24. I. M. Gelfand and S. V. Fomin, Calculus of Variations Fizmatgiz, Moscow (1961) (in Russian).

25. A. Barone and G. Paterno, Physics and Applications of the Josephson Effect, Wiley-Interscience, New-York (1982).

26. K. K. Likharev, Introduction to Dynamics of Josephson Junctions, Nauka, Moscow (1985) (in Russian). 